\title{
Observation of the drug diffusion using ultrasound contrast agent in pig meat
}

Takahiro Ando, M.D. ${ }^{1}$, Atsushi Mori, Ph.D. ${ }^{2}$, Yoko Arakawa, M.D. ${ }^{1}$, Kimitoshi Nishiwaki, M.D.,Ph.D. ${ }^{2}$

${ }^{1}$ Nagoya University Hospital, Dept of Anesthesiology, Nagoya, Japan.

${ }^{2}$ Nagoya University Graduate School of Medicine, Dept of Anesthesiology, Nagoya, Japan.

Background: The diffusion of local anesthetics in the regional nerve blockade is usually observed fluoroscopically after the injection of mixture of the drug solution and a non-ionic contrast agent lohexol. Actually, a clear contrast image of the drug diffusion can be obtained using lohexol. However, taking patient's safety into consideration, X-ray exposure in fluoroscopy is not suitable for routine use. A minimally invasive contrast agent, "Sonazoid ${ }^{\oplus}$ for Injection" (Daiichi Sankyo) consists of perflubutane microbubbles and is characterized by a prolonged ability to maintain its contrasting attributes. In the present study, to establish a simple and trustworthy method for the observation of drug diffusion, we examined the usefulness of Sonazoid ${ }^{\circledR}$ in pig meat.

Materials and methods: A mixed solution of Sonazoid ${ }^{\oplus}$ (final concentration: $1,000,3,000$, or 10,000 times dilution of clinically-used solution) and $0.04 \%$ trypan blue solution as a pigment was prepared. A nerve block needle was punctured into a pig meat and the epidural anesthesia catheter, which was filled with the prepared solution, was placed $2 \mathrm{~cm}$ from the needle tip and $0.1 \mathrm{ml}$ of the solution was injected through the catheter. After acquiring the imaging, the maximum reaching distance of the solution was measured using the contrast mode of the ultrasound diagnostic apparatus Aplio ${ }^{\mathrm{TM}}$ 300 (Toshiba Medical Systems). Then, the pig meat was sliced and the longest distance in the trypan blue stained region was measured by a ruler. The usefulness of Sonazoid ${ }^{\oplus}$ was evaluated by calculating the error rate from above two measurements. Statistical analysis was performed using the SPSS software.

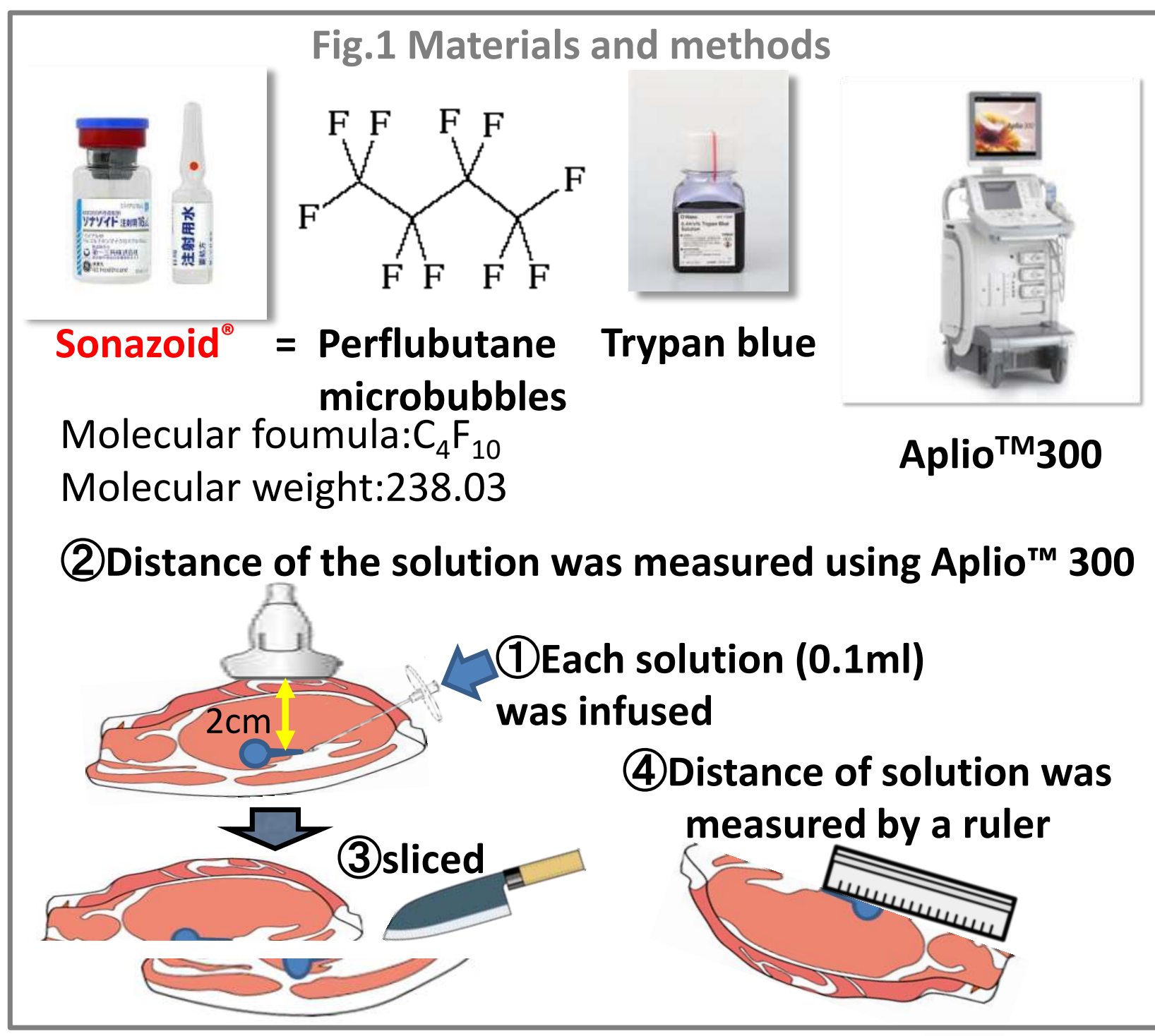

Results: The error rates of 10,000, 3,000, and 1,000 times dilution of Sonazoid ${ }^{\circledR}$ were $42.0,19.0$, and $5.0 \%$ in median, respectively $(n=5)$. The error rate of 10,000 times dilution showed a significantly higher value as compared with those of the other groups (vs. 3,000 times dilution: $p=0.015$, vs. 1,000 times dilution: $p=0.002)$. There was no significant difference of the rate between 3,000 and 1,000 times dilution $(p=0.422)$.

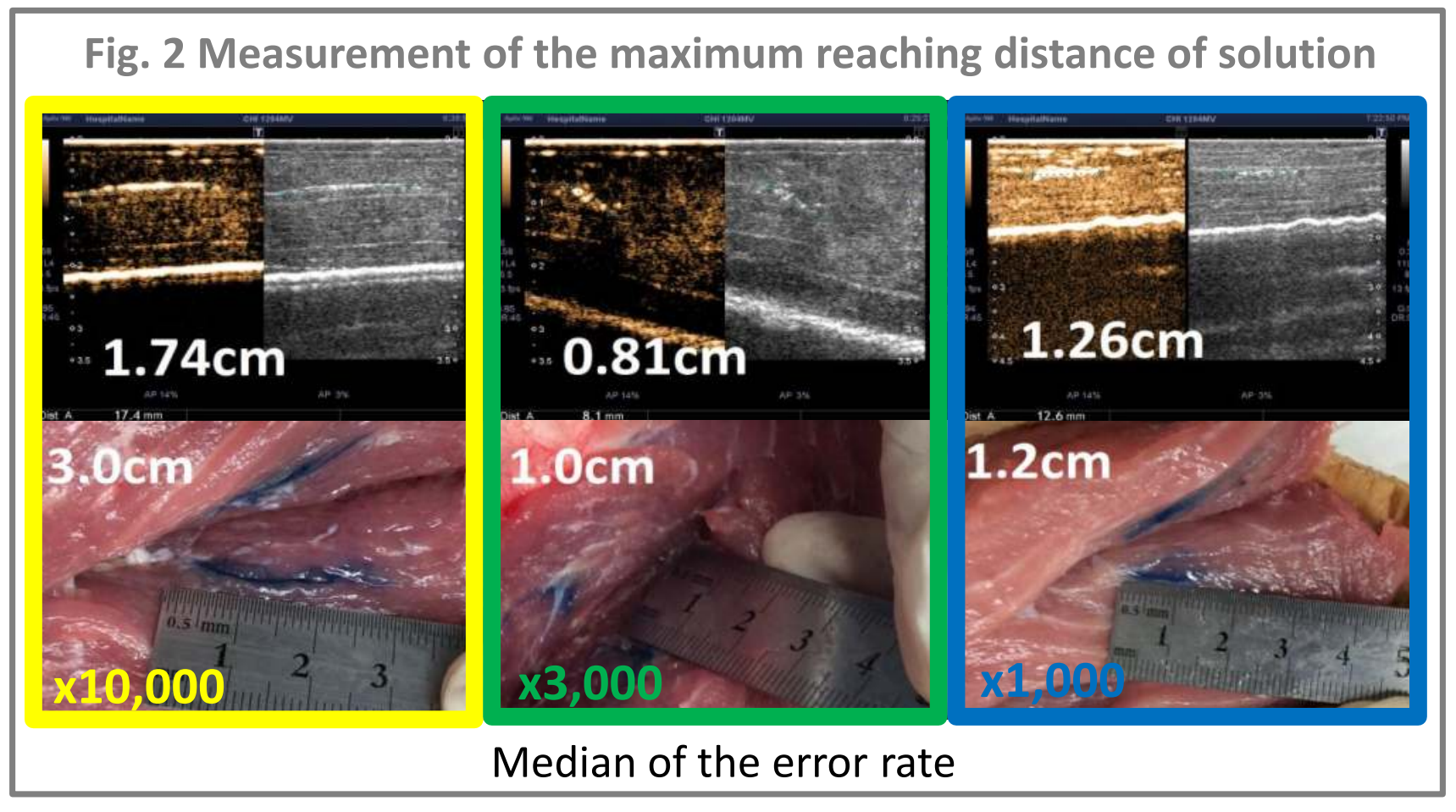

Fig. 3 Measurement of the maximum reaching distance of solution in the Echo imaging (Ei) and Trypan blue (Tb) stained region

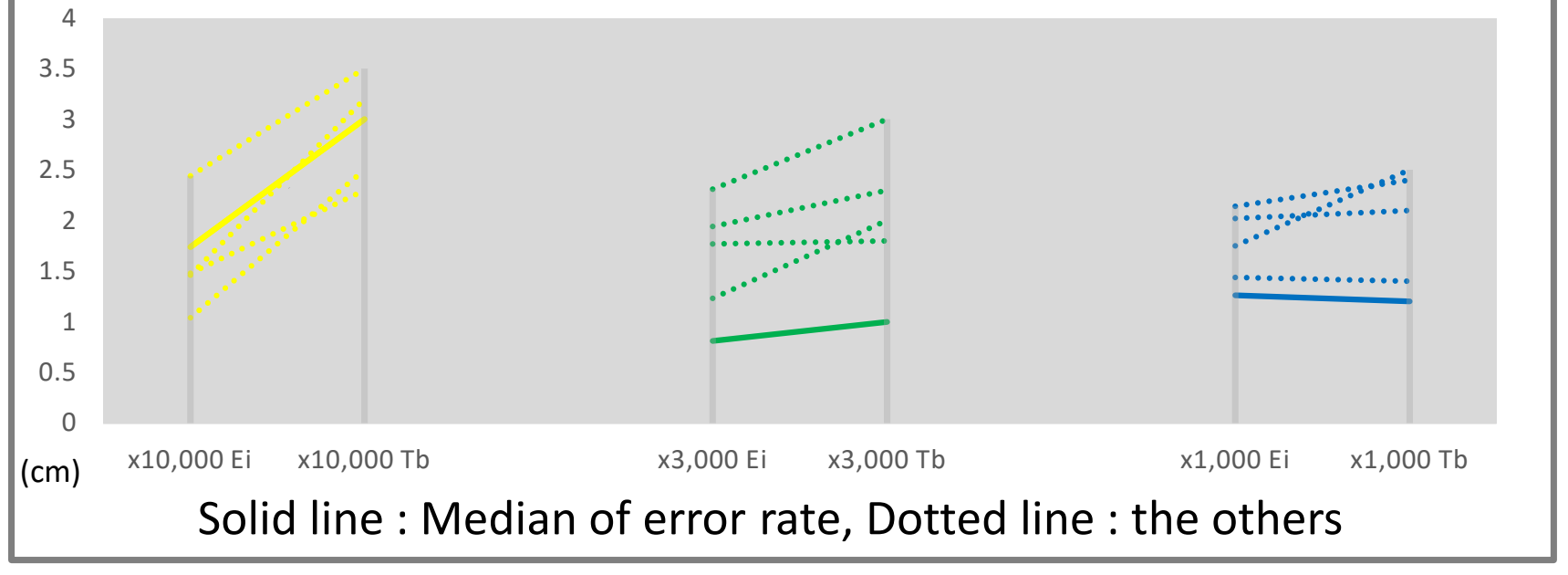

Fig.4 The error rates (\%) of $10,000,3,000$, and 1,000 times dilution of Sonazoid ${ }^{\circ}$

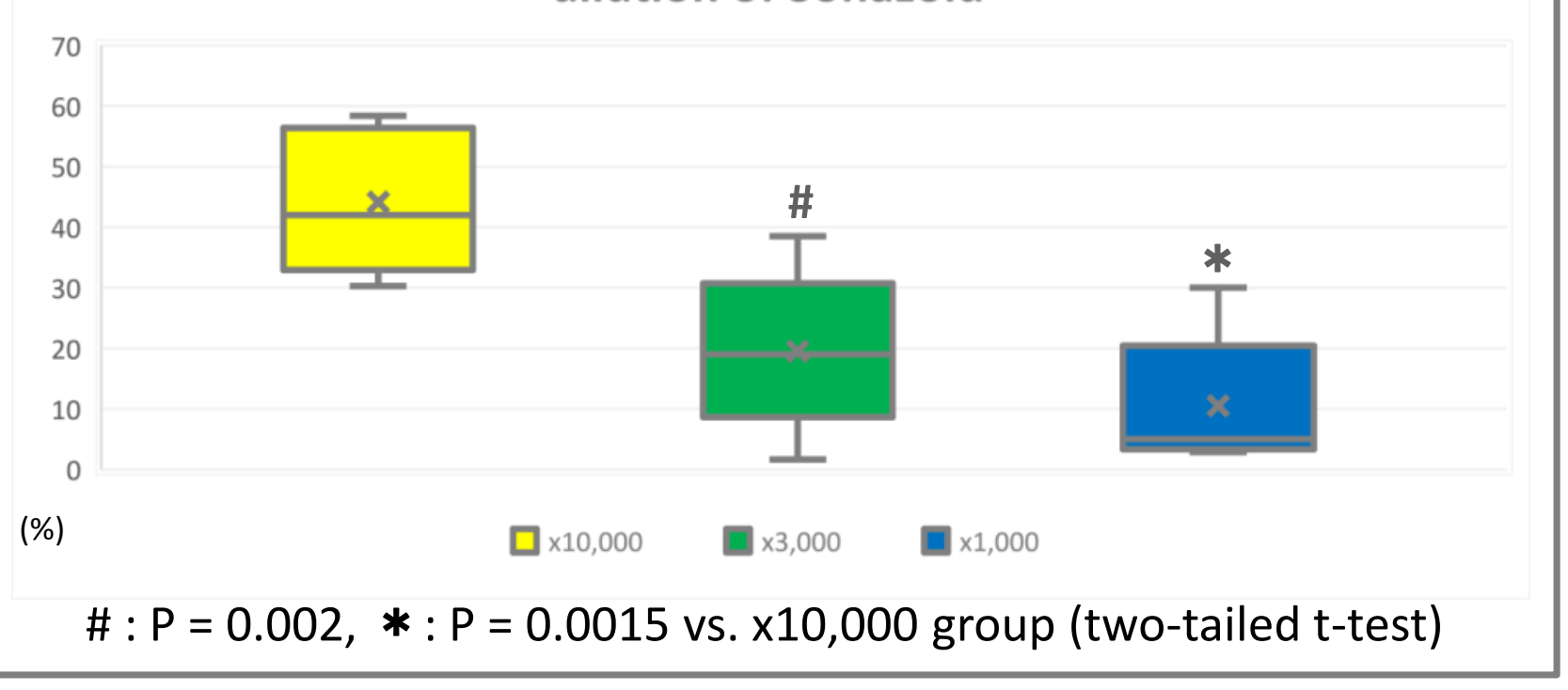

Discussion: As a limitation of this study, there is a difference in accuracy of obtained data from manual observation with ruler (minimum scale value: $0.1 \mathrm{~cm}$ ) and the ultrasonic diagnostic apparatus (minimum scale value: $0.01 \mathrm{~cm}$ ). In addition, it was difficult to measure the spreading of solution using linear probe because each sample showed a remarkably different spreading pattern. However, in the detection of distance between tips of needle and spread solution, the error rate was seemed to be very small in almost all samples of 1,000 times dilution group. Therefore, our results suggest that high

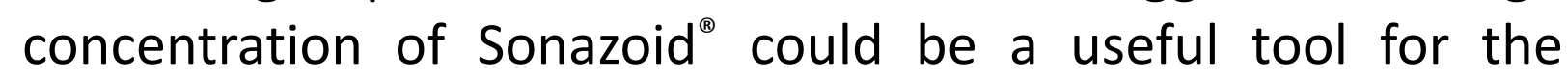
detection of drug diffusion in the regional nerve blockade.

Ultrasound contrast agent Sonazoid ${ }^{\oplus}$ is usually used for the ultrasound imaging of focal liver lesions and focal breast lesions and to help clinicians better determine whether or not a tumor is malignant or benign. Although the safety to the human body in intravenous injection has already been demonstrated, to apply this agent for detecting the diffusion of local anesthetics, we should confirm the safety with local administration.

Conclusion(s): In the present study, we demonstrated that the error rates between ultrasonic diagnostic image and the real diffusion depended on the dilution rate of Sonazoid ${ }^{\oplus}$ in the observation of liquid diffusion using pig meat. 